\title{
Design and Development of Automated Public Transportation System and Traffic Guidance System
}

\author{
B.Vamsi Krishna \\ Department of Electronics and Communication Engineering, Gudlavalleru Engineering College, Gudlavalleru
}

\begin{abstract}
As population is growing, there is an increase in the number of vehicles on the road and hence an improvement in the problems associated with traffic management, especially the Public Transport. There is also an increase in the number of accidents and various other traffic related issues. Intelligent Public Transportation Management System (IPTMS) provides the solution to most of these problems by integrating existing technologies with the underlying infrastructure. An efficient vehicle tracking system is designed and developed for tracking the movement of any equipped vehicle from any location at any time. Real time vehicle tracking for efficient transport management has become feasible with the advent of mobile technology and the omnipresence cellular network. With thus, one need not wait for so long for any on road vehicle. The ubiquity of Smart Phones and their ever increasing power at a very economical price makes them one of the most attractive options for developing IOT applications. Here, an approach based on the combination of technologies like GPS and Android is discussed which can satisfy passengers who commute by the means of public transport. The user is furnished with explicit information about the current location of nearest buses approaching the bus-stop on a mobile application. Using Android bus APP and user APP, technologies like 3G network and SMS based services in the existing mobile phones can reduce the cost and size of hardware required, as well as lead to a better output.
\end{abstract}

Keywords: GPS, GSM, Android, Web Server, IPTMS, IOT, RFID

\section{Introduction}

Public transport is a service available on sharing basis for the welfare of the general public. It includes city buses, trolley-buses and rapid transit like metro and subways. Unlike transportation modes like car-pooling, rickshaws and taxis, this system encompasses an entirety of strangers. The main reasons as to why people choose public transportation over other modes of transport are its subsidized rates, environment-friendly attributes and easy accessibility. Firstly, public transport is very economical allowing a large population to have access to it. Using a bus or a train to drive is comparatively cheaper than using a private car [1]. If people have their own car, they have to spend a lot of money on car servicing, repairs, and insurance. There are many discounts available for some individuals, like students and senior citizens who choose public transport as their transportation option to get to work or go to school. Secondly, public transport can preserve the environment by reducing the amount of pollution. With an increase in the use of public transportation, there will be a reasonable dip in the number of private vehicles on the road, therefore, improving the environment and in addition, solving the traffic congestion issues [1]. Furthermore, public transportation has good accessibility in big cities, making it easier to travel to any part of the city, making buses a favorable option to opt for. It provides personal mobility and freedom for people from every walk of life [1].

Taking into consideration the other aspects of public transportation, there are some downsides to this service as well. Public transportation, by its very nature, is far more time consuming than any other mode of transportation. Most trains and buses run in accordance with a scheduled timetable. However, these time schedules are seldom followed. There is always an uncertainty regarding the arrival of a bus. Often, buses break down causing further problem to drivers. Another difficulty we see is that public transportation often lacks organization. Drivers are often confused with regards to bus routes and bus stops. Even if the buses are running on time, they are usually crowded, the reason being, less frequency of the buses. Since the ratio of the buses to the population availing public transportation is disproportionate, overcrowded buses are not a rare sight.

With regard to all problems mentioned above, the simple knowledge of bus related information can solve a number of discrepancies related to public transportation. For instance, the time of arrival and departure of each bus, a comprehensive list of bus-stops, etc. can prove to be very constructive. Hence, an Intelligent Public Transport Management system is necessary to solve these issues. In order to provide necessary bus data to all passengers, this paper proposes a Smart Public Transport System where all relevant information of the bus will be gathered, processed, and presented to the user. This system includes introducing an hardware device inside the bus for indicating the approaching bus terminals and the remaining bus route. Moreover, the system helps in nullifying the long waiting hours at bus stops. Along with the uncertainty in time, there is also an apprehension regarding the capacity of a bus [2].Even if the passenger is aware about the arrival time of the bus, 
they do not know how many additional people can be accommodated inside the bus. The information will be half-baked and hence of no use. Thus, determining capacity of any given bus is equally important to the arrival time estimation. Therefore, by using high-end technologies, like Android and NFC code facility, and integrating them with the existing system, we can provide a good solution to the issues discussed

[2]. To summarize, the system will eradicate the uncertainty in arrival time that drivers face every day and prove to be of great assistance in planning their journeys well in advance.

The paper is organized as explained further. Section II provides a detailed literature survey on tracking in public transportation using RFID and GPS as well as the older hardware approaches. Section III goes on to give a brief description of the proposed architecture. Section IV presents an android base implementation of the proposed architecture. Section V and VI put light on the conclusion and the future scope of this architecture.

\section{Literature Survey}

With the advent of advanced technologies a lot of countries have started investing in their transportation sector. As a result, extensive research has been carried out in the past few years. The state-of-the art advancements in wireless communication and universal Internet capabilities have encouraged the development of Intelligent Public Transportation Management System. In this section we will survey in brief about the work being done in Information and Communication Technologies (ICT) usage in public transport towards tracking and scheduling.

\section{A. GPS in Public Transportation}

Automated Fare Collection (AFC) System also known as the Transit Smart Card System provides us an edge over the manual fare collection system by lowering labor costs and also increasing the efficiency of manual fare collection process. The desire to extract more information than just a simple deduction of fare from transit smart cards has led to the research efforts in extracting other relevant information such as points of origin where a passenger would board a bus and have the data recorded as the passenger's smart card is scanned. To achieve this, a Markov chain based Bayesian decision tree algorithm has been developed in this study, where in the algorithm is verified with the use of public transportation vehicles that are equipped with GPS tracking and data loggers. Conclusively, it is stated that data collected to represent points of origin when a passenger's transit smart card is scanned, is crucial to the process of transit system planning [4]. In another research the GPS technology is being utilized towards tracking and scheduling of buses. This has been implemented in Ahmedabad India, where the government has deployed a GPS- enabled Bus Rapid Transit System (BRTS) solution to solve their transportation issues in a sustainable fashion. Introduction of the BRTS was motivated by the need for increased reliability and security with prime focus on reducing travel time. The tracking and scheduling of all buses on all routes is controlled centrally at an integrated control center [5].

\section{B. RFID in Public Transportation}

RFID technology mainly includes three components: RFID Tag, RFID Reader and Middleware logic for interaction with the back-end database continuously. Many applications for RFID technology have been suggested over the past few years. However, these efforts have been challenged with regard to their feasibility, deployment, privacy, security and such other aspects.

Other micro controllers like Arduino Mega [10] and Raspberry Pi are also being used for the development of vehicle tracking applications on a large scale .These boards provide an easy user-friendly development environment to create effective applications in a simple manner. One such research is focused on applying RFID towards potential passengers passing through a simulated bus door equipped with commercial off-the-shelf RFID readers and antennas, passengers as they board and exit the bus.

The research concluded that RFID technology can be effectively used for this type of application; however, there was an issue which prevent the concept. It was found that in case of no line of sight between smart cards and reader in accordance with the radiation pattern and positioning of antenna, there might be some performance glitches as these factors are very crucial and critical to the whole process of recognition [6]. Research was also focused on how RFID Technology can be used to solve trouble faced by Public Transport authorities especially in metropolitan cities by doing research for enhancement of automated tracking of buses that can be very useful in providing useful estimates regarding bus arrival times and in turn provide improved passenger convenience. A real time tracking and monitoring system is employed which utilizes a framework of Event, Condition and Action (ECA). This proves to be helpful in filtering data effective to remove all the unwanted or inaccurate details and then categorize useful data by clustering. Utilization of collected data for prediction of bus movement is also discussed in an effort to improve the tracking system and in turn enhance traveling experience by Public Transport [7]. 


\section{Hardware Based Access}

Many hardware based approaches have been proposed to solve the problem of bus tracking and scheduling. Most of the approaches use the same basic architecture: Micro controller, GPS module, GPRS/GSM Modem. All these are bundled together to form an On-Board Unit (OBU) which is fitted onto the bus. The GPS collects location data which is sent to the micro controller which in turn sends it to the GPRS/GSM Modem, transmitting data through wireless cellular network to the back-end server. The back-end server is responsible for processing all the incoming data and extracting meaningful information from it like dynamic route tracking. Pham, Micheal, Chi [8] suggest a novel vehicle tracking system consisting of the GPS module, which is used to obtain the vehicle's coordinate and the GSM modem, which is used to transmit the location to the user's phone through the mobile network. The Global System for Mobile Communications (GSM) is the 2G digital cellular mobile network [9]. It is widely used around the world. Although improvements to GSM such as the next generation systems have been rolled out to supply for faster data centric traffic, backward compatibility to GSM is still maintained. Due to its wide availability, it is chosen as the medium for transfer of location information.

The simple and inexpensive Short Message Service (SMS) allows users to send data. For the purpose of this project, the SMS is more than sufficient for sending the location information. The hardware components used by Pham, Micheal, Chi [8] in their implementation include the state-of-the-art u-blox NEO-6Q GPS receiver module and u-blox LEON-G 100 GSM module. A micro controller, the Arduino Uno [10] is also employed to control both modules and to provide an easily customizable platform for any required application. Other micro controllers like Arduino Mega [10] and Raspberry Pi are also being used for the development of vehicle tracking applications on a large scale .These boards provide an easy user-friendly development environment to create effective applications in a simple manner.

\section{Proposed System}

Hardware approaches to IPTS are too dependent on the quality of the sensors and the micro controllers used. Moreover flexibility of such approaches is also less along with the complexity involved in the bundling together of the various components into a single hardware framework. The hardware based methods also suffer from problems due to electrical noise, maintenance, wear and tear etc.

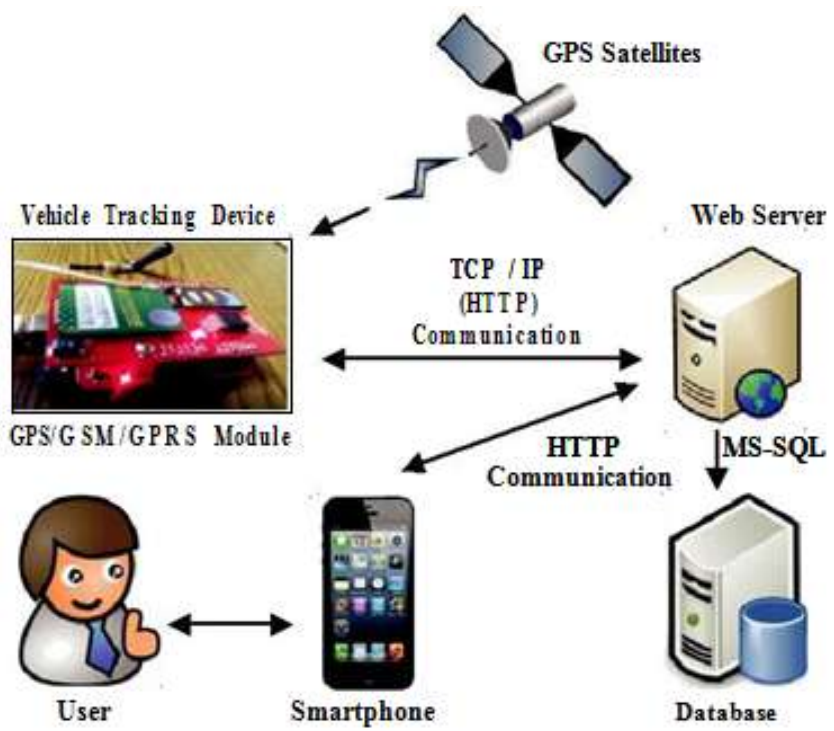

Fig. 1. Block Diagram showing whole architecture of the system

Keeping in mind all these problems, here proposing a simple Android and IOT based approach which can provide dynamic bus tracking information to the bus stops as well as the drivers in an efficient manner. The architecture which is basically divided into three parts. The On Board Module (OBM) consists of an Android Smart phone equipped on the bus, basically used to track the position of the bus using the inbuilt GPS of the phone. The collected location information is then sent to a server using $3 \mathrm{G}$ network of the phone. The back-end consists of a server module which receives continuous data from the OBM. The received data is used to extract information which is then used to service various queries. The user module consists of an Android application as well as a SMS based system. It can be used by drivers to track the location of the bus in real-time environment as well as plan their travel in advance.

Apart from this here also proposing installation of NFC codes on each bus-stop. NFC code proves to be an effective measure to provide information about the passengers regarding travelling information. Whether 
they have allowed to travel or not. The proposed architecture is clearly shown in Fig.1. with complete block diagram. It provides further insight into the working of the complete system as a whole.

\section{Implementation Using Android}

In this implementation a proposed concept using Android OS. Android Studio version 2.2 [11] is used to test on a Android Phone supporting minimum SDK 4.4 (Kit Kat) with API level 19 and also used ASP.NET with C\# software for the web server.

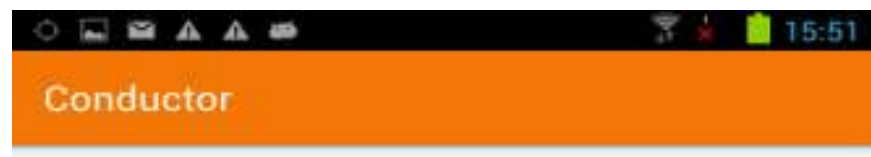

\section{Name : MSN Farookhi}

\section{Department : ECE}

Mobile NO : 1234567895

\section{Location Name: Vijayawada Bus Stand}

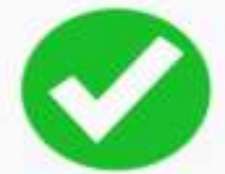

$10 / 08 / 2017$

\section{Address}

No

\section{↔ 可}

Fig.2. Android activity showing user authentication details.

\section{A. On Board Module}

Connecting separate GSM/GPRS modules together to form an integrated unit is a tedious task. It can take advantage of the fact that these components are already inbuilt in our Smart Phones. Given the fact that most people now a days use Smart Phones and that they are available at a reasonable price, makes this approach viable. Here proposed an application based on Android OS utilizing the inbuilt GPS to track the location of the bus continuously and push it to a server on a frequent basis.

Once the application is started for the first time, an interactive activity [17] pops up asking for the detailed description of the particular bus route. Each and every bus is given particular unique identification number and specific names or numbers to source, destination stations and every bus stop in particular route. This is a onetime activity for the duration of the entire application. Once all the fields are filled the information is stored permanently in the database. Next time when the application is started it opens up another activity which simulates the events in case the bus actually starts moving on the roads. The Android activity shows in Fig 3. the available bus stops between source and destination. The current location information is fetched progressively from GPS receiver and updated the current location data to the user. 


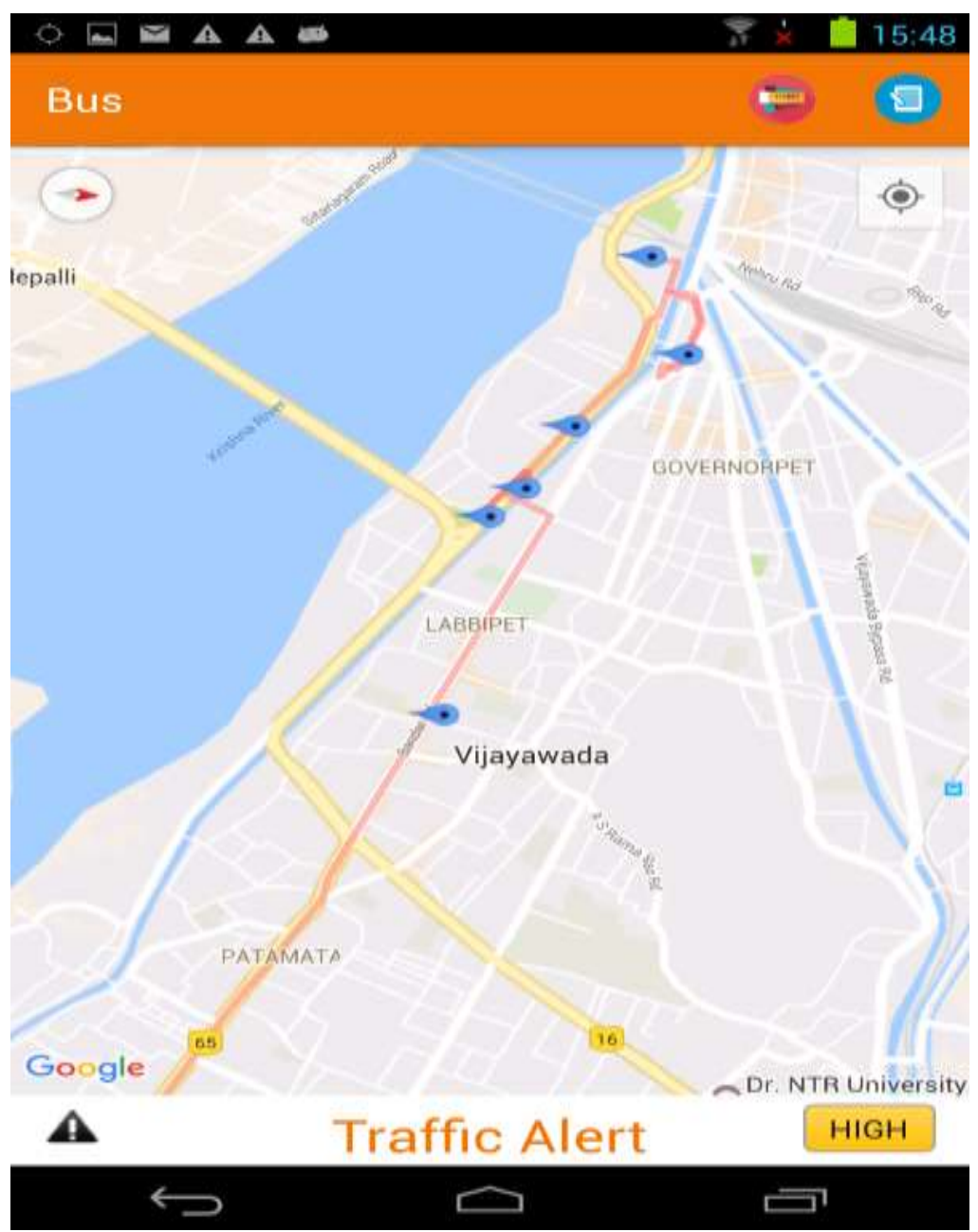

Fig. 3. Android Activity showing bus stop terminal in bus module

The application can be reset by the driver by clicking on reset button after the bus reaching destination. After this the application begins tracking the route of the bus again in a similar manner as described above. The Smart Phone can be powered by using the $12 \mathrm{~V}$ battery. Since there are problems related to usage of GPS due to high battery usage and inability to use indoors, so here proposing a combination of NETWORK PROVIDER and GPS PROVIDER service of Android OS. This system will dynamically switch between both providers according to need. Along with this we can also take advantage of recently launched off line mode of Google Maps.

The previous coordinates are compared with the current coordinates to determine whether bus is stationary or not. Accordingly the longitude and latitude values are used to calculate the distance of the bus from the terminals and the expected time of arrival which is then sent to the server.

\section{B. Server Module}

The Web Server module forms the core of this proposed system. It serves as the back-end tool. The server contains the information about the routes of the bus as well as the intermediate bus stops on the route. The server also processes request from a user regarding the location of the bus at a particular bus stop. The server maintains a database of information related to the bus, routes and stops in the form of tables. The server database can be defined in many ways, to scale down memory requirement, improve access speed, or reduce the number of queries. It can be used MS-SQL SERVER [18] in this implementation. The Android application installed on the bus sends information to the server in JSON [20] encoded format which is stored in the tables created on the server using MS-SQL [19]. As soon as the information reaches the server using WEBSOCKET Technology, which is responsible for updating the information and is send to the user. 

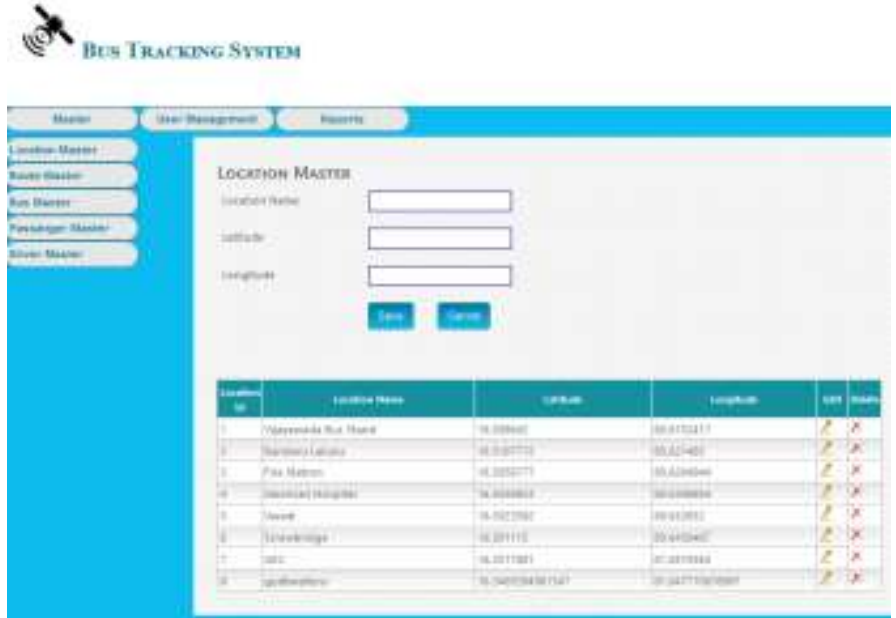

Fig. 4. Bus route locations stored in database design using MS-SQL Server

As the bus is moving on its desired route the GPS receiver gets the information about the bus from satellites and this data send to the web server through TCP/IP communication protocol. Server constantly updates the information about the moving bus left from source station and next station columns in the tables so that if a user queries about the availability of a bus at a required bus stop , using the data in the tables appropriate response can be generated and provided to the inquiring user. The server is multi-threaded in nature and hence can process multiple simultaneous user requests at a time. As soon as a user request comes in, the server creates a thread to serve that user. The thread is then responsible for handling all communications with that particular user. After the communication is over the thread goes away from the pool.

\section{User Module}

Although a lot of change in the Public Transport zone, the communication gap between a regular user and the information generated from the system remains curtail. My implementation is aimed towards unite this gap. An application proposed on the user side which can collect the data about moving bus in a particular route and exact position of the bus terminal. As a result the uncertainty and long waiting hours at the bus stop is completely avoided to the users by this approach. Fig 5 shows the passenger's details stored in the database which is designed using MS-SQL. It includes the particulars like passenger ID, name, mobile number, NFCID, location name, NFC code, expiry date and E-mail ID.
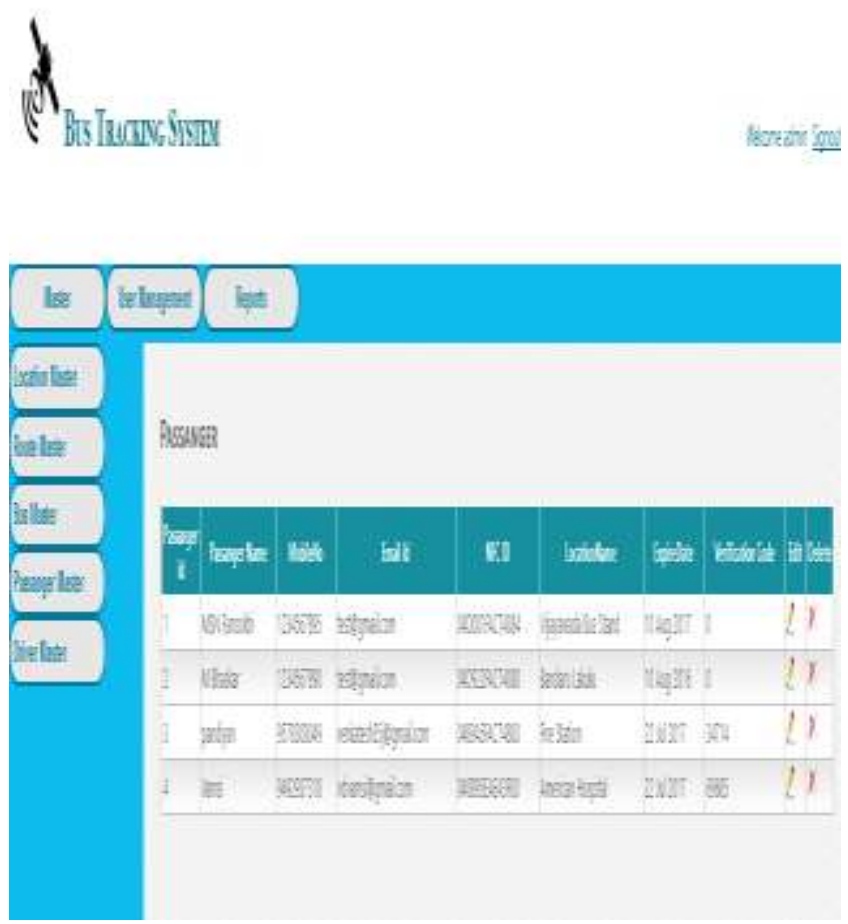

Fig. 5. User details stored in database design using MS-SQL Server 
The user simply enquires about the availability of the bus at a particular bus-stop through the Mobile application and the server responds by sending appropriate reply within a small amount of time. Fig 6 shows the android activity of the current bus location on the user mobile display which is specified in the web server, between source and destination. At the same time it can also proposed a SMS gateway which can be used by the commuters in case of unavailability of internet or Smartphone. The user can send a SMS enquiring about the availability of the bus and appropriate reply in the form of an SMS will sent back to the user. In addition to this it can also accommodate NFC codes to every user and authenticate the user to travel in the bus. With these codes one can avoid misuse of bus services.

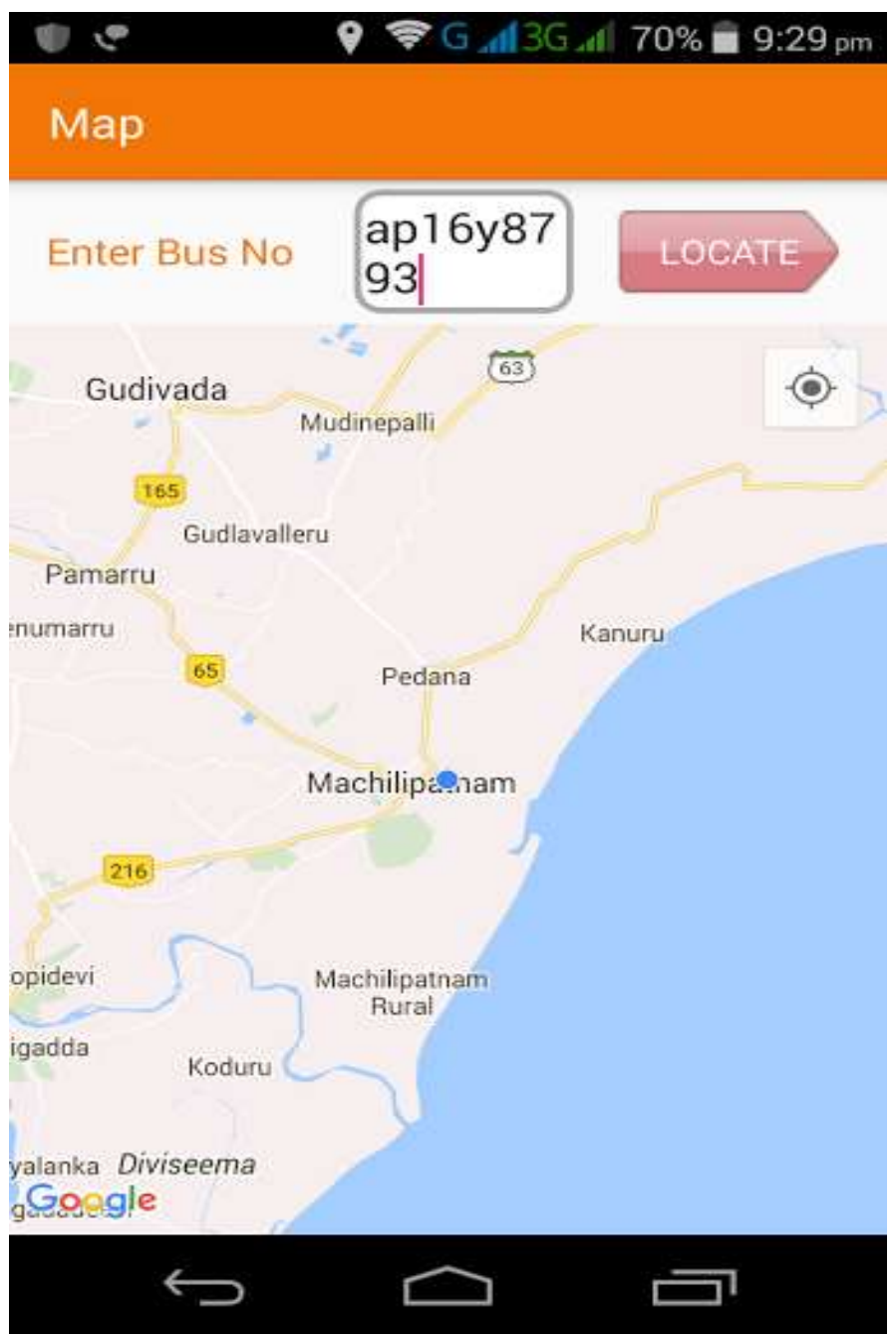

Fig. 6. Android activity showing bus location in the user Mobile

\section{Conclusion}

In this view the traffic management system on the road not as an individual problem, but as a global issue which needs to be accepted in a discreet manner. This paper given an efficient structure of IPTM System that dynamically tracks the location of the bus and update the position to the users. The measurements are updated at regular intervals, every time the bus module sends an update to the server. It distributes this information on demand, to passengers who send request using a smart phone application. The issues with traffic can be constrained as more people will opt for the efficient and economical public transportation as a medium of travel on a frequent and regular basis. With the information on demand service, the drivers can plan their journey well in advance, hence saving a lot of time and making the individual more productive. The commotion of the next arriving bus terminal is also solved by the in bus display module which will give details of the route at regular intervals. To conclude, this proposed system assists commuters, users and also the administrators of the transport system in a very convenient manner. 


\section{Future Extension}

The improvement in internet is vast and their useful operation could lead to major finding in the field of IOT. In future, it can be realize simple cashless system to regulate the capabilities of the NFC present in the smart phones. This cashless method of payment can give us a count of the number of passengers present on board, which could be used further to estimate the capacity of the bus. These details could also be transmitted to the waiting passengers so that they can plan their journey accordingly. The number of people requesting for the bus details can be further used to decide the frequency of the buses, thereby reducing the confusion and making it a good experience for the drivers.

\section{References}

[1]. 2015 American Public Transportation Association. Available online at : http://www.apta.com/mediacenter/ptbenefits/Pages/default.aspx .

[2]. SUN Wen-xia and SONG Ti , ZHONG Hai, "Study on Bus Passenger Capacity Forecast Based on Regression Analysis Including Time Series", 2009 Int. Conf. on Measuring Technology and Mechatronics Automation, 2009, IEEE, DOI 10.1109/ICMTMA.2009.268.

[3]. Sleyman Eken and Ahmet Sayar, "A Smart Bus Tracking System Based on Location-Aware Services and QR Codes", Innovations in Intelligent Systems and Applications (INISTA) Proceedings, 2014 IEEE International Symposium, Alberobello, DOI:10.1109/INISTA. 2014.68736 34.

[4]. Xiao-Lei, M et al (2012), Transit Smart Card Data Mining for Passenger Origin Information Extraction, I Journal of Zhenjiang University Science C, Vol.13(10), pp.750-760,DOI: 10.1631/jzus.C12a0049.

[5]. Oberli, $\mathrm{C}$ et al., Performance Evaluation of UHF RFID Technologies for Real Time passenger Recognition in Intelligent Public transportation Systems, IEEE Trans. Intell Transp. Syst.,Vol.1 1(3), pp.748-753.DOI: 10.1109/TITS.2010.2048429.

[6]. Menzes, B et al (n.d).Challenges in RFID Deployment- A Case Study in PublicTransportation, Availablefrom http://citeseerx.ist.psu.edu/viewdoc/downloaddoi=10.1.1.104.3449\&rep =rep1\&type=pdf.

[7]. Pham Hoang Oat, Micheal Drieberg and Nguyen Chi Cuong. Development of Vehicle Tracking System using GPS and GSM Modem, 2013 IEEE Conference on Open Systems(ICOS),December24,2013,Sarawak,Malaysia,DOI:10.1109/ICOS.2013.6735054.

[8]. A. EI-Rabbany, GPS for Vehicle Navigation in Introduction to GPS: The Global Positioning System,Norwood, MA: Artech House, 2006.

[9]. SeokJu Lee, Girma Tewolde, Jaerock Kwon "Design and Implementation of Vehicle Tracking System Using GPS/GSM/GPRS Technology and Smartphone Application" Internet of Things (WFIoT), 2014 IEEE World Forum on April 2014, 14255652, 10.1109/WF IoT.2014.6803187.

[10]. P. Widhalm, P. Nitsche, and N. Brandie, Transport mode detection with realistic smart phone sensor data, in Proc. IEEE 21st ICPR, Tsukuba, Japan, 2012, pp. 573576.

[11]. Y. J. Byon, B. Abdulhai, and A. Shalaby, Real-time transportation mode detection via tracking global positioning system mobile devices, J. Intell. Transp. Syst., 13, no. 4 pp. 161170, 2009.

[12]. S. Reddy et al., Using mobile phones to determine transportation modes, ACM Trans. Sens. Netw., vol. 6, no. 2, p. 13, Feb. 2010.

[13]. X. Yu et al., Transportation activity analysis using smart phones, in Proc.IEEE CCNC, 2012, pp. 6061.

[14]. Mark Smith, "Intelligent Mobile Application for Traffic Monitoring”, ITNG, 2015, 2015 12th International Conference on Information Technology - New Generations (ITNG), 2015 12th International Conference on Information Technology - New Generations (ITNG) 2015, pp. 621-625, doi:10.1109/ITNG.2015.103.

[15]. Arduino. (2013). Arduino: Main Page [Online]. Available: http://www.arduino.cc.

[16]. Android Studio 1.0 Developers Blog Available: http://androiddevelopers.blogspot.in /2014/12/android-studio-10.html.

[17]. AndroidDevelopersAvailable:http://developer.android.com/reference/aandroid/app/Activity.html..

[18]. https://www.mssqltips.com/sqlservertip/948/sql-server-websites/

[19]. MS-SQL: The World's most popular open source database Available: https://www.mssql.com/.

[20]. JSON Available: http://www.json.org/

[21]. Web Socket: https://www.websocket.org/aboutwebsocket.html.

[22]. GPS: http://www.gsmarena.com/glossary.php3?term=gps.

[23]. Web server application: http://asp.net-tutorials.com/

[24]. PäiviJaring, ViliTörmänen, ErkkiSiira, and TapioMatinmikko, "Improving Mobile Solution Workflows and Usability Using Near Field Communication Technology", Technical Research Center of Finland Oulu, Finland, Springer-Verlag Berlin Heidelberg, pp. $358-373,2007$ 